\title{
La productividad de los puertos en la región del APEC: un estudio a través del análisis de la frontera estocástica
}

\author{
The productivity of ports in APEC region: A study through the stochastic \\ frontier analysis
}

\author{
Odette Virginia Delfín-Ortega ${ }^{*}$, César Lenin Navarro-Chávez \\ Universidad Michoacana de San Nicolás de Hidalgo, México
}

Recibido el 9 de abril de 2018; aceptado el 7 de noviembre de 2019

Disponible en Internet el: 5 de marzo de 2020

\section{Resumen}

Esta investigación tiene como objetivo determinar la Productividad Total de los Factores (PTF) de los 40 principales puertos de la región del APEC durante el periodo 2005-2015. Se mide la PTF a partir de sus componentes: cambio tecnológico, cambio en la eficiencia técnica y cambio en la eficiencia de escala, instrumentando el Análisis de la Frontera Estocástica (SFA). Se tiene como variable dependiente el número de teus y como variables independientes la longitud del muelle y el personal ocupado. En promedio los puertos del APEC tuvieron un incremento en su PTF del 5.10\% durante los años de estudio, donde el aumento en la eficiencia de escala es quien más incidencia tiene con un $4 \%$.

Código JEL: C51, L91, O33

Palabras clave: APEC; Frontera estocástica; Productividad total de los factores; Puertos

\footnotetext{
*Autor para correspondencia Correo electrónico odettedelfin@umich.mx (O. Delfín Ortega).

La revisión por pares es responsabilidad de la Universidad Nacional Autónoma de México. 


\begin{abstract}
This research aims to determine Total Factor Productivity (TFP) of 40 main ports of the APEC region during the period 2005-2015. TFP is measured from its components: technological change, efficiency technical change and scale efficiency change, instrumenting Stochastic Frontier Analysis (SFA). The number of teus is the dependent variable and the length of dock and personnel employed as independent variables. On average, the APEC ports had an increase in their TFP of 5.10\%, where scale efficiency change is the one with the highest incidence with $4 \%$.
\end{abstract}

JEL codes: C51, L91, O33

Keywords: APEC; Stochastic frontier; Total factor productivity; Ports

\title{
Introducción
}

El Foro de Cooperación Económica Asia-Pacífico (APEC), es una región integrada por 21 economías: Australia, Brunei, Canadá, Indonesia, Japón, Corea del Sur, Malasia, Nueva Zelanda, Filipinas, Singapur, Tailandia, Estados Unidos, República de China, Hong Kong, Taiwán, México, Papúa Nueva Guinea, Chile, Perú, Rusia y Vietnam. En el año 2014, sus participantes representaron en conjunto el 54\% del Producto Interno Bruto (PIB) del mundo, el $50 \%$ del comercio internacional y el 40\% de la población del planeta (APEC, 2015).

El Foro de Cooperación Económica de Asia-Pacífico (APEC por sus siglas en inglés) tiene su origen en la primera reunión celebrada en Camberra Australia el 6 de noviembre de 1989, donde los cuatro puntos de su agenda fueron: a) desarrollo económico mundial y regional; $b$ ) liberalización del comercio mundial, el rol de la región Asia-Pacífico; c) oportunidad para la cooperación regional en áreas específicas; y, d) pasos futuros para la cooperación económica Asia-Pacífico (APEC, 1989). Es decir, las economías integrantes del APEC se agrupan para alcanzar objetivos comunes que los llevan a constituirse como región.

La pertinencia de este estudio en las economías de Asia-Pacífico, se debe a que la participación de esta región en el comercio marítimo ocupa el primer lugar a nivel mundial; y el APEC reconoce que el servicio de transporte marítimo es vital para aumentar la competitividad comercial (APEC, 2016).

La importancia estratégica de la infraestructura y los servicios de transporte marítimo para el acceso al mercado, la producción globalizada y la competitividad comercial es clave para el desarrollo de un país, por lo que el APEC cuenta con el Grupo de Trabajo de Transporte de APEC (TPT-WG) quien trabaja para mejorar la conectividad y profundizar la integración económica regional mediante la promoción de conectividad de infraestructura de calidad; mejorando la accesibilidad, seguridad, resiliencia, eficiencia y sostenibilidad del transporte; y mejoramiento socioeconómico transversal (APEC, 2019). 
Hoy en día, los puertos buscan la manera de reducir los tiempos de movilización de carga al mejorar la eficiencia de sus operaciones. El uso más eficiente de las instalaciones portuarias, junto con las mejoras en la escala de operaciones, permite mejorar la productividad total de los factores y por lo consiguiente elevar la competitividad del transporte marítimo. Todos estos elementos dan la pauta a la necesidad de realizar estudios que permitan elevar la productividad de los puertos de las economías del APEC y esto impacte en su dinamismo económico.

La industria marítima de Asia Pacífico tiene un 39\% de participación en todo el mercado global (IMO, 2015). Debido al elevado flujo comercial que hay en la región, la operación marítima es una parte fundamental dentro de su comercialización y de acuerdo al World Shipping Council (2017), los puertos que presentaron mayor movilización de contenedores en el año 2015, se encuentran en la región del APEC: China, Singapur, Corea, Hong Kong y Japón. Sin embargo, México no ha logrado posicionarse entre los puertos más competitivos a nivel mundial y de acuerdo al índice global de competitividad realizado por el Foro Económico Mundial (World Economic Forum, WEF) (2017), México en el año 2016 ocupaba el lugar 57 en el rubro de infraestructura portuaria y para el año 2017 descendió al lugar 62. Lo que da cuenta de la necesidad de estudios, propuestas e inversión que logren elevar su productividad y potencializar así el sistema portuario, impactando de manera directa en el desarrollo económico del país. La productividad puede mejorarse aún más, con un mayor uso de la tecnología existente, como grúas pórtico, automatización y software para incrementar el flujo de tráfico y almacenaje, pues factores como, los tiempos de espera en los muelles y la disponibilidad de grúas y equipos son determinantes en la eficiencia (Sánchez et al., 2015).

En el caso de México actualmente está en una etapa de crecimiento portuario debido a la expansión del comercio exterior que hay a nivel mundial (UNCTAD, 2018), y uno de los objetivos de la Dirección General de Puertos (2016) es impulsar el desarrollo de los puertos en condiciones de competitividad, calidad y eficiencia, así como también mejorar el aprovechamiento del valor de los espacios portuarios para elevar la disposición de recursos destinados a nuevas inversiones que permitan fomentar el desarrollo de los negocios en los puertos mexicanos. Por lo que se plantea la necesidad de realizar estudios que identifiquen la productividad portuaria en nuestro país, comparándola con los principales puertos de la región más dinámica a nivel mundial que es el APEC, lo cual contribuirá en la implementación de políticas públicas orientadas al establecimiento de puertos marítimos mexicanos de clase internacional.

Este estudio considera el periodo de 2005 al 2015 debido a que de acuerdo a la Conferencia de la Naciones Unidas sobre Comercio y Desarrollo (UNCTAD por sus siglas en inglés), en el año 2015, el crecimiento del sector portuario experimentó notables reducciones, específicamente los fletes marítimos de carga contenerizada disminuyeron continuamente durante el año 2015, hasta llegar a niveles mínimos. En un esfuerzo por detener la caída de los fletes y 
reducir las pérdidas, los diferentes actores del sector, así como investigadores han estudiado diversas medidas para mejorar la eficiencia y optimizar las operaciones (UNCTAD, 2016).

El crecimiento del comercio de mercancías también ha sido débil en relación con el crecimiento del PIB mundial, esto debido a la debilidad de la demanda mundial, la desaceleración de la actividad económica y también a factores estructurales como la fragmentación de las cadenas de suministro (UNCTAD, 2017).

Las necesidades de los puertos han cambiado en los últimos veinte años, ya que antes la preocupación giraba solo en torno al apoyo de servicios marítimos. Sin embargo, actualmente se busca que los puertos sean plataformas logísticas que articulen diversos medios de transporte, almacenadoras, industrias y una mayor conectividad hinterland y foreland (García, 2018).

La industria portuaria depende de factores exógenos y endógenos. Los factores exógenos corresponden a la desaceleración del comercio mundial. Por otro lado, los factores endógenos, en el sector público corresponden a: gobernanza portuaria y logística y a la administración de los procedimientos burocráticos, tales como aduanas, sanidad, migración, etc. Los factores endógenos en el sector privado son los que pertenecen a cada compañía portuaria y a toda su operacionalización (industria portuaria) (Sánchez y Lara, 2016).

Los problemas en la industria portuaria se acentúan debido:

a. A la intensidad de los fenómenos y a los cambios ocurridos en ambos factores tanto exógenos como endógenos;

b. A la confluencia de todos ellos simultáneamente.

Dentro del sector portuario privado es importante destacar que la propia actividad portuaria donde actualmente hay más fusiones y adquisiciones, trae como consecuencias, la presión por baja en las tarifas y por mayor velocidad en el manejo de contenedores. Lo que ha tenido como consecuencia también el acortamiento del ciclo de vida de las inversiones portuarias y una cierta caída de la productividad, menores rendimientos corporativos y baja en las proporciones de los valores de venta de las terminales (Sánchez y Lara, 2016).

La organización del sector del transporte marítimo tiene un profundo impacto sobe el volumen de comercio, los costes de transporte y la competitividad económica (UNCTAD, 2016). En los puertos en general, existen diversos grupos de interés que impulsan la demanda de indicadores de desempeño. Los responsables políticos necesitan investigaciones basadas en datos concretos, los inversores necesitan medios para reflejar el rendimiento y los gestores portuarios necesitan una base comparativa práctica para llevar a cabo la evaluación comparativa y la planificación estratégica.

La UNCTAD (2015), menciona que el rendimiento de los puertos y terminales es importante porque influye en la competitividad comercial de un país. Los factores determinantes del rendimiento de los puertos y terminales son muchos: las relaciones laborales, la cantidad 
y el tipo de equipo para la manipulación de la carga, la calidad de la zona de transbordo, los canales de acceso al puerto, el acceso desde tierra, la eficiencia de las aduanas, así como las posibles concesiones a los operadores internacionales de terminales.

Los puertos marítimos internacionales operan como facilitadores del comercio en la economía mundial. Son instrumentos estratégicos de la política comercial en las economías nacionales y representan un nexo de unión importante entre naciones mediante la circulación eficiente y rentable de las mercancías, personas e información (Sánchez y Lara, 2016).

Conocer el comportamiento de la productividad de un puerto es importante para adoptar las medidas que permitan corregir las ineficiencias detectadas y mejorar las fortalezas del puerto (Kim y Sachis, 1986). Para González y Trujillo (2006) los puertos buscan maximizar la transferencia de carga, y consideran que la capacidad de manipulación de la mercancía de un puerto depende de su productividad.

Diversos autores han analizado la productividad portuaria a través de diferentes metodologías. En el caso del cálculo de la PTF a través del índice Malmquist, Schøyen y Odeck (2017), miden los cambios de la productividad a través de este índice de los principales puertos de Noruega y Reino Unido durante 2009-2014. Los principales hallazgos muestran que, la productividad aumentó en aproximadamente $0.6 \%$ por año para todos los puertos considerados. Nwanosike, Tipi, y Warnock-Smith (2016), realizaron un estudio de la PTF con esta misma metodología de los puertos de Nigeria en el periodo 2000-2011, para comparar el crecimiento de la productividad antes y después de la reforma portuaria del país. Baran y Górecka (2015), calcularon la eficiencia y la productividad también con este índice de los principales puertos internacionales en el periodo 1996-2012. Mokart y Shah (2013), miden la productividad de los principales puertos de contenedores de Malasia utilizando en la medición de la PTF el índice Malmquist con rendimientos constantes y variables, obteniendo resultados significativos en ambos enfoques. Por su parte Halkos y Tzeremes (2012), calculan la PTF de los puertos griegos en el periodo 2006-2010, incorporando además, la técnica bootstrap. Borafull (2010), calcula la PTF del sistema portuario español en el periodo 1990-1999. En los resultados se encuentran, que los puertos han tenido mejoras en la productividad, y el progreso técnico fue quien la determinó en mayor medida, específicamente a partir de 1997. Por su parte Fu, Song y Guo, (2009), analizan la PTF de 10 terminales de contenedores de los puertos de China en el periodo 2001-2006, mediante el índice Malmquist también. Cheon, Dowall, y Song (2009), calculan la productividad de los puertos en el ámbito internacional para el periodo 1991-2004 con el índice Malmquist. Otros estudios han calculado la productividad a través de métodos paramétricos, tal es el caso de Chang y Tovar (2014), que obtienen la eficiencia y la PTF de los puertos peruanos y chilenos a través del Análisis de Frontera Estocástica (SFA) en el periodo 2004-2010. En la misma dirección De (2006), realizó un estudio sobre la PTF en los principales puertos de la India utilizando la metodología SFA. Por su parte Lightfoot, 
Lubulwa y Malarz (2012), con mínimos cuadrados ordinarios calcularon la PTF de los puertos de Australia en los años 1997-2010.

Específicamente estudios sobre la productividad en la región del APEC se han realizado por diversos autores como son Yen-Chun, Chih-Hung, Goh y Yung-Hsiang (2016), quienes utilizaron la metodología Malmquist para comparar la productividad portuaria de los países desarrollados y los países en desarrollo en el APEC, desagregándola en cambio de eficiencia de escala, cambio de eficiencia técnica y el cambio tecnológico. Los resultados indican que la tasa promedio de capacidad utilizada entre los puertos de los miembros de APEC fue de solo $65.7 \%$ durante 2002-2011 y el factor que más afectó la productividad fue el cambio en la eficiencia de escala.

En las mediciones de eficiencia del APEC se encuentran Kutin, Thuy y Valléec (2017), que realizaron un estudio sobre eficiencia utilizando la metodología DEA de 50 puertos de la región de ASEAN utilizando un modelo CRS. Los hallazgos pueden ayudar a los administradores de puertos en la región de la ASEAN a tomar decisiones sobre si aumentar el tráfico de contenedores. Chun-Chu (2008), hace un estudio sobre eficiencia utilizando la metodología DEA aplicando un modelo CCR y un modelo BCC en el periodo 1998 -2001, en 10 puertos de la región de Asia y el Pacífico. Los resultados muestran que en promedio, la eficiencia estimada con el modelo CCR es la más baja.

Hsuan-Shih y Ming-Tao (2005), calculan la eficiencia de los puertos seleccionados de la región de Asia - Pacífico trabajando con la metodología DEA para el año de 1996, obteniendo en general resultados de eficiencia elevados en la mayor parte de los puertos.

En esta investigación se tiene como objetivo determinar la Productividad Total de los Factores (PTF) de las 40 terminales de contenedores de los principales puertos de las economías del Foro de Cooperación Económica Asia-Pacífico (APEC), durante el periodo 2005-2015, a través del Análisis de la Frontera Estocástica (SFA). La pregunta de investigación que se plantea es: ¿Qué factores determinaron la Productividad Total de los Factores durante el periodo 2005-2015? La hipótesis a considerar es que es el cambio en la eficiencia de escala quien determina la Productividad Total de los Factores en los puertos de la región del APEC para el periodo 2005-2015. El trabajo está estructurado en seis apartados. En el primero, se encuentra la introducción; en el segundo, se abordan los fundamentos teórico-metodológicos, desarrollando la metodología del Análisis de Frontera Estocástica para obtener la Productividad Total de los Factores; en el tercero, se presenta el desarrollo del modelo; en el cuarto, se encuentran los resultados; mientras que, en el quinto apartado, se tiene la discusión de resultados; finalmente, en el sexto se exponen las conclusiones. 


\section{Fundamentos teórico-metodológicos}

En este apartado se analizan las bases teóricas y metodológicas de la Productividad Total de los Factores. Se empieza por definir la productividad, entendiéndola como la razón entre la cantidad producida y los insumos utilizados. Mientras que, la Productividad Total de los Factores es la razón de la producción neta con la suma asociada de los factores de insumos de mano de obra y capital (Sumanth, 1994). Existen diversas técnicas para medir el cambio en la Productividad Total de los Factores, las más comunes se pueden dividir en 3 principales categorías (Girales 2013):

a. Números Índices. Diewert (1976), define los números índices como aquellos que pueden ser derivados de alguna función de producción subyacente, señala también que los índices más utilizados son los de Laspeyres, Paasche, Fisher y Törnqvist. Cada uno de esos índices usan una forma funcional diferente para agregar las diversas entradas y resultados del proceso de transformación. En esta técnica únicamente es necesario conocer los datos de los insumos y productos en medidas únicas (índices) en función de sus precios relativos, utilizando diversas fórmulas de números índices.

b. Funciones de distancia no paramétricas. Estos enfoques manejan el proceso de agregación basado directamente en la cantidad de información sobre inputs y outputs y un mínimo de supuestos sobre la forma general de la tecnología (es decir, el proceso de transformación). Se basan en el cálculo de cocientes de funciones de distancia, obtenidas mediante programación lineal. Permite medir el crecimiento de la PTF que experimenta cualquier unidad que transforme factores productivos, así como descomponer ese crecimiento en cambio tecnológico y cambio de eficiencia técnica. Las funciones de distancia más comunes para medir el crecimiento de la productividad se basan en la noción del índice de productividad de Malmquist, que fue introducido como un concepto teórico por Caves, Chirstensen y Diewert (1982). Más tarde Färe et al. (1992) demostraron cómo se puede estimar el índice de productividad de Malmquist mediante el Análisis de la Envolvente de Datos (DEA).

c. Enfoques econométricos. Similar a las funciones de distancia no paramétricas, los enfoques econométricos también pueden estimar un índice de productividad utilizando solo información sobre cantidades de inputs y outputs y un conjunto de mínimas suposiciones, principalmente sobre la forma general de la tecnología y la distribución del ruido aleatorio y el término de ineficiencia. Los enfoques econométricos también pueden descomponer la Productividad Total de los Factores en sus componentes: cambio tecnológico, cambio en la eficiencia técnica y de escala. Los enfoques econométricos más comunes son: Mínimos Cuadrados Ordinarios Corregidos (COLS) o, el modelo de Análisis de Frontera Estocástica (SFA). 


\section{El modelo de Solow}

La teoría del crecimiento de Solow, en la que el patrón de crecimiento de la productividad refleja el llamado progreso tecnológico (es decir, el residuo de Solow), ha sido la primera metodología determinista propuesta para estimar la PTF y se ha utilizado para estimar esta productividad tanto a nivel agregado como sectorial. Solow (1957), fue quien contribuyó a establecer la Productividad Total de los Factores como un concepto operacional, a partir de la función de producción. En su artículo «Technical change and the aggregate production function», publicado en 1957, describe el modelo basado en la teoría de la producción, específicamente en la curva de Cobb Douglas cuya forma estándar considera dos factores de producción: capital y mano de obra.

El método del residuo de Solow considera la PTF como una variable no observable en forma directa, que representa la parte de la producción que no puede ser explicada por los actores productivos utilizados. El método del residuo establece que para calcular la PTF es necesario hacer un supuesto respecto de la función de producción, es decir, utilizar una aproximación matemática a la forma en que se combinan los factores en la producción de bienes y servicios para, posteriormente, estimar el residuo que representará a la PTF. El residual de Solow se basa en la función de producción Cobb Douglas con rendimientos de escala constantes y calcula el cambio tecnológico neutral " o "Hicks-neutral” lo que implica que se incrementen por igual las variables del capital y del trabajo. El método del residuo de Solow (1957), indica que $\mathrm{Q}$ representa la salida mientras que $\mathrm{K}$ y $\mathrm{L}$, representan las entradas de capital y trabajo en "unidades físicas", por lo que la función de producción agregada se puede escribir como:

$$
\mathrm{Q}=\mathrm{F}(\mathrm{K}, \mathrm{L} ; \mathrm{t}) \text {. }
$$

Solow (1957) menciona que el cambio técnico es neutral, es decir que un desplazamiento de la función de producción no altera la distribución del ingreso para una relación capital-trabajo dado. En ese caso, la función de producción adopta la forma especial.

$$
Q=A(t) f(K, L)
$$

Se diferencia la ecuación 2 totalmente con respecto al tiempo y se divide entre Q y se obtiene:

$$
\frac{\dot{\mathrm{Q}}}{\mathrm{Q}}=\frac{\dot{\mathrm{A}}}{\mathrm{A}}+\mathrm{A}\left(\frac{\partial \mathrm{f}}{\partial \mathrm{K}}\right) *\left(\frac{\dot{\mathrm{K}}}{\mathrm{Q}}\right)+\mathrm{A}\left(\frac{\partial \mathrm{f}}{\partial \mathrm{L}}\right) *\left(\frac{\dot{\mathrm{L}}}{\mathrm{Q}}\right)
$$

Los puntos indican las derivadas respecto al tiempo. Ahora se define $\mathrm{W}_{\mathrm{k}}=\left(\frac{\partial Q}{\partial K}\right) *\left(\frac{K}{Q}\right) \mathrm{y} \mathrm{W}_{\mathrm{L}}$ $=\left(\frac{\partial Q}{\partial L}\right) *\left(\frac{L}{Q}\right)$ las formas relativas del capital y del trabajo, y se sustituyen en la ecuación 3 
(nota que $\partial \mathrm{Q} / \partial \mathrm{K}=\mathrm{A} \partial f / \partial \mathrm{K}$, etc.) y de ahí el resultado:

$$
\frac{\dot{\mathrm{Q}}}{\mathrm{Q}}=\frac{\dot{\mathrm{A}}}{\mathrm{A}}+\mathrm{w}_{\mathrm{k}}\left(\frac{\dot{\mathrm{K}}}{\mathrm{K}}\right)+\mathrm{w}_{\mathrm{L}}\left(\frac{\dot{\mathrm{L}}}{\mathrm{L}}\right)
$$

Entonces la tasa del progreso tecnológico en dos periodos está dado por:

$$
\frac{\Delta \mathrm{A}}{\mathrm{A}}=\frac{\Delta \mathrm{Q}}{\mathrm{Q}}-\mathrm{w}_{\mathrm{k}} *\left(\frac{\Delta \mathrm{K}}{\mathrm{K}}\right)-\mathrm{w}_{\mathrm{L}} *\left(\frac{\Delta \mathrm{L}}{\mathrm{L}}\right)
$$

Donde:

$\frac{\Delta A}{A}=$ Tasa de crecimiento del progreso técnico.

$\frac{\Delta Q}{Q}=$ Tasa de crecimiento del producto.

$\frac{\Delta K}{K}=$ Tasa de crecimiento del stock de capital.

$\frac{\Delta L}{L}=$ Tasa de crecimiento del stock de trabajo.

$w_{k} y w_{L}=$ Remuneraciones del capital y del trabajo.

Algunos autores como Summer (1986), Hall (1988, 1990), Mankiw (1989), Evans (1992), Chen (1997), Zheng, Liu y Bigsten (1998), Kumbhakar y Lovell (2000), Songqing, Hengyun, Huang, Ruifa y Rozelle (2009), han argumentado que el residuo de Solow tiene varios errores: mencionan que es muy limitante por ser solo un enfoque contable de las fuentes de crecimiento y que la estimación del cambio tecnológico no es adecuada calcularla solamente en presencia de rendimientos constantes a escala debido a las imperfecciones propias del mercado, otra de las críticas que mencionan es que se interpretan erróneamente las mejoras en la productividad atribuidas solamente al progreso técnico. Sin embargo, esta suposición solo es válida si las empresas son técnicamente eficientes, operando así en sus fronteras de producción y aprovechando todo el potencial de la tecnología, lo cual no siempre sucede. Por lo tanto, el progreso tecnológico no puede ser la única fuente de crecimiento de la productividad, y será posible aumentarla por el mejoramiento de la eficiencia técnica. Mencionan también que la medición de la PTF con el residual de Solow, solo permite calcular el cambio tecnológico de manera neutral en términos de Hicks. Sin embargo, el cambio tecnológico a menudo no es neutral, ya que en muchas ocasiones algunos factores de producción se benefician más que otros; además, algunos grupos adoptan nuevas tecnologías antes que otros. Limitarse a un solo tipo de cambio tecnológico no refleja una realidad de los efectos competitivos del mercado. 


\section{Medición de la PTF a través de métodos paramétricos}

Una forma alternativa de analizar el crecimiento de la PTF es desagregándolo en sus componentes: progreso tecnológico y mejora de la eficiencia. Siguiendo el enfoque común en el análisis de frontera estocástica (SFA), suponemos que las ineficiencias pueden generar una brecha entre la producción real y la frontera de producción, dado el estado actual de la tecnología.

En este marco, el progreso tecnológico (representado por una tendencia temporal) desplaza la frontera de producción hacia arriba para todos los estados, mientras que una mejora en la eficiencia técnica mueve a los estados hacia la frontera de producción (Cardarelli y Lusinyan, 2015). Los primeros autores que empezaron a utilizar enfoques paramétricos fueron Aigner y Chu (1968), Seitz (1971), Timmer (1971), Afriat (1972), Richmond (1974). Sin embargo, son las aportaciones de Aigner, Lovell y Schmidt (1977) y Meeusen, Van Den Broeck (1977) con las que se desarrolla la conceptualización de la frontera estocástica, a partir de la cual se consolida la referencia metodológica de esta línea de estudio sobre la eficiencia, en la que se parte de una función de comportamiento eficiente, sea de producción o de costos.

$$
\mathrm{y}=\mathrm{X} \beta+\varepsilon
$$

$Y=$ Es el vector de los outputs.

$\beta^{\prime}=$ Es un vector de los parámetros a estimar.

$\mathrm{X}=$ Es el vector de todos sus inputs.

$\varepsilon=$ Es el término estocástico de perturbación.

Donde se asume que el término de error tiene dos componentes $\varepsilon=v-u$ para las funciones de producción, y $\varepsilon=v+u$ para las funciones de costos.

Más adelante Kumbhakar y Lovell (2000), realizaron la medición de la Productividad Total de los Factores utilizando el análisis de frontera estocástica, descomponiéndola en cambio tecnológico, cambio en la eficiencia técnica y cambio en la eficiencia de escala.

$$
T F P_{i t}=T E_{i t}+T P_{i t}+(E-1) \sum_{j} \frac{E_{j}}{E} x_{j} \quad \mathrm{j}=1,2
$$

Donde:

El $T F P_{i t}=$ Representa el cambio de la PTF.

$T E_{i t}=$ Representa el cambio en la eficiencia técnica.

$T P_{i t}=$ Es el cambio tecnológico.

$E_{j}(\mathrm{j}=1,2)=$ Representa la elasticidad del output con respecto a cada input (capital y trabajo). $(E-1) \sum_{j} \frac{E_{j}}{E} x_{j}=$ Es el componente de la eficiencia de escala. 
Estimación y descomposición

Una función de producción de frontera estocástica se puede definir por (Kumbhakar y Lovell, 2004):

$$
y_{i t}=f\left(x_{i t}, t, \beta\right) \exp \left(v_{i t}-u_{i t}\right)
$$

Donde yit es el vector del output de la firma $(\mathrm{i}=1,2, \ldots . \mathrm{N})$ en el periodo $\mathrm{t}(\mathrm{t}=1,2, \ldots \mathrm{T})$.

La frontera de producción estocástica tiene dos partes: determinística y estocástica; $\mathrm{f}(\mathrm{xit}, \mathrm{t}, \beta)$, es la parte determinística de la frontera de producción con el vector del parámetro de tecnología $\beta$ a ser estimado, xit es el vector de los inputs, $t$ es una tendencia de tiempo; $v$ es la parte estocástica, la cual captura los efectos aleatorios, -u es el término de ineficiencia técnica. Las formas funcionales translogarítmica y Cobb-Douglas son las formas más comunes encontradas en la literatura económica aplicada al momento de representar funciones de producción. Los autores recomiendan una serie de criterios que permiten discriminar entre las distintas formas funcionales a utilizar, observan que, mientras ambas son similares en términos de linealidad en los parámetros y regularidad, la translogarítmica es superior en flexibilidad (Coelli et al., 2005). Para fines de esta investigación, se calcula el cambio en la Productividad Total de los Factores con la metodología del Análisis de Frontera Estocástica, desagregando el cambio en la eficiencia técnica y el cambio tecnológico de un modelo para datos panel con una especificación translogarítmica de la función de producción propuesto por Kumbhakar y Lovell, (2004).

$$
\begin{gathered}
\ln y_{i t}=\beta_{0}+\sum_{n} \beta_{n} \ln x_{n i t}+\beta_{t} t+\frac{1}{2} \sum_{n} \sum_{j} \beta_{n j} \ln x_{n i t} \ln x_{j i t}+\frac{1}{2} \beta_{t t} t^{2}+ \\
\sum_{n} \beta_{n t} \ln x_{n i t} t+v_{i t}-u_{i t}
\end{gathered}
$$

Donde:

$\mathrm{y}_{\mathrm{it}}=$ Representa el output observable en el periodo t.

$X_{\text {it }}=$ Es el conjunto de factores de producción.

$t=$ es un componente de tendencia temporal,

$\beta=$ son los parámetros desconocidos a ser estimados.

$v_{i t}=$ es el componente de error de ruido aleatorio.

$u_{i t}=$ es el componente de error de la ineficiencia técnica. 
Modelo de frontera de producción de datos de panel

Los modelos de datos panel para medir la eficiencia con econometría los iniciaron Pitt y Lee (1981) y Jondrow, Materov, Lovelland y Schmidt (1982), Schmidt y Sickles (1984), Battese y Coelli (1988), en donde la eficiencia era tomada como invariante y constante en el tiempo. Posteriormente, Cornwell, Schmidt y Sickles (1990), Kumbhakar (1990), fueron los primeros autores en proponer una frontera de producción estocástica para un modelo de datos panel con la eficiencia variante en el tiempo y continuaron Battese y Coelli (1992) trabajando bajo este mismo esquema.

Kumbhakar (1990) desarrolló la siguiente función paramétrica de tiempo:

$$
\beta(t)=\left[1+\exp \left\{\gamma t+\delta t^{2}\right\}\right]^{-1}
$$

El modelo de Kumbhabar contiene dos parámetros adicionales que deben de ser estimados con máxima verosimilitud $\gamma$ y $\delta$ La función $\beta(t)$ satisface las propiedades $0 \leq \beta(t) \leq 1 \mathrm{y} \beta(t)$ y puede incrementar o decrecer monotónicamente y ser cóncava o convexa dependiendo de los signos y magnitudes de los dos parámetros $\gamma$ y $\delta$ tanto para el modelo de datos de panel de efectos fijos como variables.

Battesi y Coelli (1992) por su parte proponen el modelo para la estimación de la eficiencia variante en el tiempo utilizando máxima verosimilitud para estimar todos los parámetros:

$$
\beta(t)=\exp \{-\gamma(t-T)\}
$$

Aquí, el parámetro desconocido representa la tasa de cambio en la ineficiencia técnica, y los efectos de ineficiencia técnica en periodos anteriores son una función exponencial determinista de los efectos de ineficiencia en el período final.

El cambio en la eficiencia se representa de acuerdo a Kumbhakar y Lovell, (2004) de la siguiente manera:

$$
\Delta T E=\widehat{u_{\imath}} \cdot \widehat{\gamma} \cdot \exp \{-\hat{\gamma}(t-T)\}
$$

Donde:

$\widehat{u_{\imath}}=$ Es el estimador para el cálculo de la ineficiencia,

$\exp \{-\hat{\gamma}(t-T)\}=$ La eficiencia técnica variente en el tiempo.

$\hat{\gamma}=$ Parámetro para ser estimado

$(t-T)=$ Es el efecto del tiempo. 
La interpretación del componente del cambio en la eficiencia es que se captura el movimiento de la unidad $i$ hacia la frontera, cuando el resultado es mayor a cero $\Delta T E>0$ significa que hubo una mejora en la eficiencia en el tiempo, por el contrario cuando el resultado es menor a cero $\Delta T E>0$ es que empeoró la eficiencia en el tiempo y cuando el resultado es igual a cero $\Delta T E=0$, las unidades no mostraron ningún cambio en el periodo analizado.

\section{Cambio tecnológico}

El cambio tecnológico está asociado al desplazamiento de la frontera de posibilidades de producción del sector o la industria a la que pertenece. La siguiente función translogarítmica involucra, además del efecto independiente de t, la interacción entre el término de tendencia y los insumos de producción a través del cual se puede calcular el cambio tecnológico no neutral (Kumbhakar y Lovell, 2004). Donde el output es asumido en función de dos inputs: capital (K) y trabajo (L).

$$
\begin{gathered}
\ln y_{i t}=\beta_{L} \ln L_{i t}+\beta_{K} \ln K_{i t}+\frac{1}{2} \beta_{L L}\left(\ln L_{i t}\right)^{2}+\frac{1}{2} \beta_{K K}\left(\ln K_{i t}\right)^{2}+\beta_{L K}\left(\ln L_{i t}\right)\left(\ln K_{i t}\right)+ \\
\beta_{t} t+\frac{1}{2} \beta_{t t} t^{2}+\beta_{L t}\left(\ln L_{i t}\right) t+\beta_{K t}\left(\ln K_{i t}\right) t+v_{i t}-u_{i t} \\
\Delta T C_{i t}=\frac{\partial \ln f\left(x_{i t}, t ; \beta\right)}{\partial t}=\hat{\beta}_{t}+\hat{\beta}_{t t} t+\hat{\beta}_{L t} L_{i t}+\hat{\beta}_{K t} K_{i t}
\end{gathered}
$$

El progreso tecnológico está representado por la derivada de la función de producción en relación con el tiempo. El cambio tecnológico $\Delta T C_{i t}$, puede ser positivo o negativo, lo que refleja que se desplaza hacia arriba o hacia abajo la frontera de producción. Cuando el resultado es mayor a cero $\Delta T C_{i t}>0$ significa que hubo progreso tecnológico, por el contrario cuando el resultado es menor a cero $\Delta T C_{i t}>0$, es que hubo un retroceso tecnológico y cuando el resultado es igual cero $\Delta T C_{i t}=0$, la frontera permanece constante durante los períodos analizados y por lo tanto la tecnología no tuvo ningún cambio.

\section{Desarrollo del modelo}

En esta investigación se presenta un modelo para datos panel donde se tiene una función de producción translogarítmica con la especificación de Kumbhakar y Lovell (2004). Se calcula el cambio de la Productividad Total de los Factores desagregándola en cambio tecnológico, 
cambio en eficiencia técnica y cambio en eficiencia de escala para el periodo 2005-2015 utilizando la metodología del Análisis de Frontera Estocástica (SFA).

La muestra de estudio se basa en las terminales portuarias de contenedores de la región del APEC, donde se identifican aquellas que tuvieron una movilización de más de un millón de teus anuales en el año 2015, de acuerdo al reporte de World Shipping Council y al Port Industry Statistics - American Association of Port Authorities. La selección se integró por 40 puertos, como se muestra en la tabla 1.

Tabla 1

Puertos de la región del APEC, 2015

\begin{tabular}{|c|c|c|c|}
\hline & País & Puerto & Millones de Teus \\
\hline 1 & Australia & Melbourne & 2.63 \\
\hline 2 & Australia & Sidney & 2.33 \\
\hline 3 & Canadá & Metro Vancouver & 3.05 \\
\hline 4 & China & Guangzhou & 17.22 \\
\hline 5 & China & Qingdao & 17.47 \\
\hline 6 & China & Shenzhen & 24.20 \\
\hline 7 & China & Tianjin & 14.11 \\
\hline 8 & China & Dalian & 9.45 \\
\hline 9 & China & Xiamen & 9.18 \\
\hline 10 & China & Ningbo & 20.63 \\
\hline 11 & China & Lianyungung & 5.01 \\
\hline 12 & China & Suzhou & 5.10 \\
\hline 13 & China & Yingkou & 5.92 \\
\hline 14 & China & Shanghai & 36.54 \\
\hline 15 & Corea & Busan, & 19.45 \\
\hline 16 & Corea & Inchon & 2.36 \\
\hline 17 & Corea & Kwangyang & 2.32 \\
\hline
\end{tabular}




\begin{tabular}{|c|c|c|c|}
\hline 18 & Estados Unidos & Long Beach & 7.19 \\
\hline 19 & Estados Unidos & Los Angeles, U.S.A. & 8.16 \\
\hline 20 & Estados Unidos & Okland- Sn Francisco & 2.27 \\
\hline 21 & Estados Unidos & Seattle-Tacoma & 3.53 \\
\hline 22 & Filipinas & Manila & 4.23 \\
\hline 23 & China & Hong Kong & 20.07 \\
\hline 24 & Indonesia & Tanjung Priok, Jakarta, & 5.20 \\
\hline 25 & Indonesia & Tanjung Perak, Surabaya & 3.12 \\
\hline 26 & Japón & Tokio & 7.52 \\
\hline 27 & Japón & Osaka & 4.93 \\
\hline 28 & Japón & Nagoya & 2.631 \\
\hline 29 & Malasia & Port Kelang & 11.89 \\
\hline 30 & Malasia & Tanjung Pelepas & 9.10 \\
\hline 31 & México & Manzanillo & 2.45 \\
\hline 32 & México & Lázaro Cárdenas & 1.05 \\
\hline 33 & Perú & Callao & 1.9 \\
\hline 34 & Singapur & Singapur & 30.92 \\
\hline 35 & Tailandia & Laem Chabang & 6.82 \\
\hline 36 & Tailandia & Bankgok & 1.55 \\
\hline 37 & Taiwán & Kaohsiung, & 10.26 \\
\hline 38 & Taiwán & Keelung & 2.66 \\
\hline 39 & Vietnam & Ho Chi Minh & 5.31 \\
\hline 40 & Vietnam & Haiphong & 3.87 \\
\hline
\end{tabular}

Fuente: World Shipping Council y AAPA, 2017. 


\section{Descripción de variables}

Para poder calcular el cambio en la Productividad Total de los Factores y sus componentes, se identifican las variables con las que se va a trabajar. Se empieza realizando una revisión literaria para elegir las metodologías y variables aplicadas en el sector portuario (véase tabla 2).

Tabla 2

Revisión de literatura

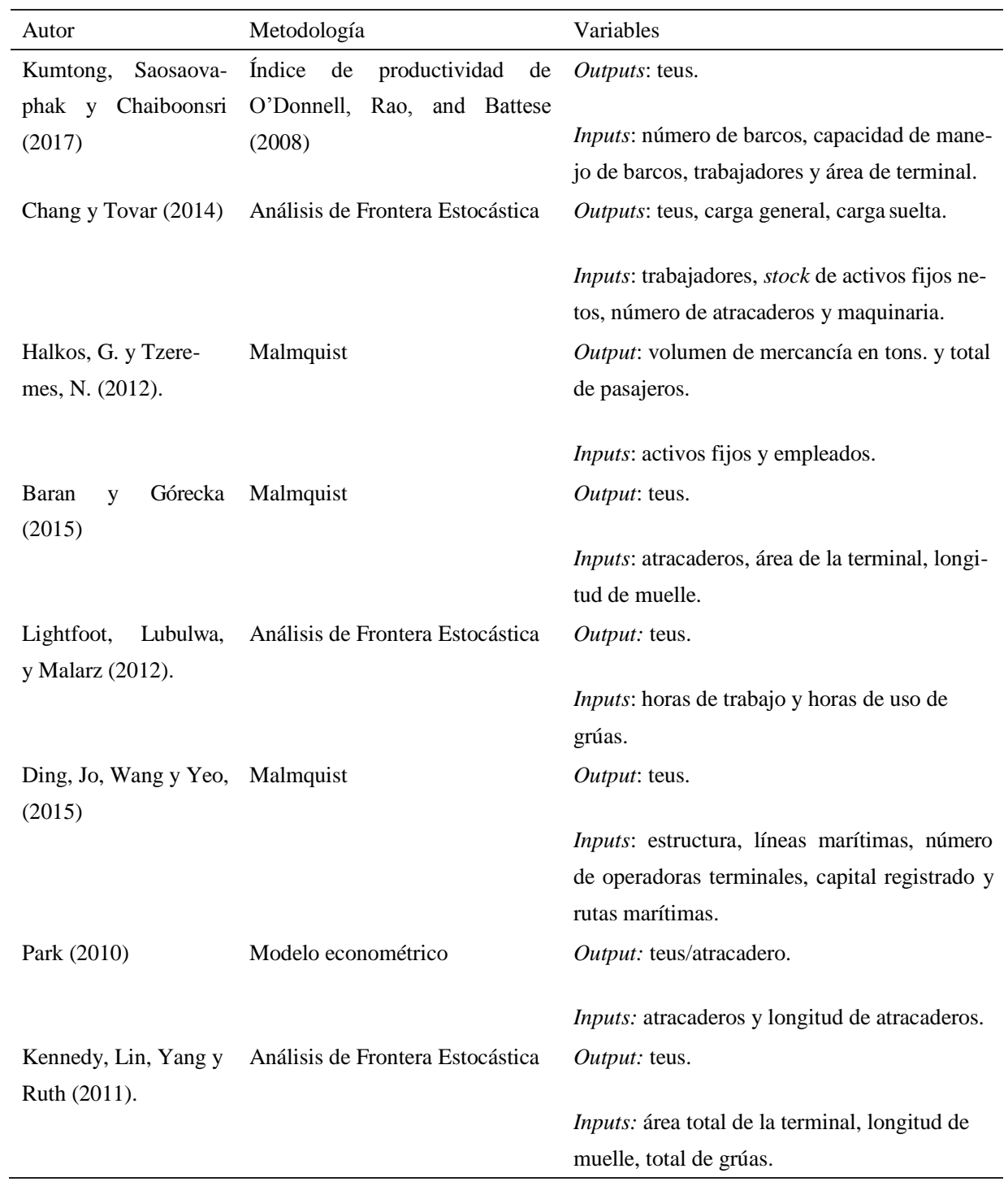

Fuente: Elaboración propia con base en la literatura revisada. 
En este estudio, la selección de variables se realizó en función de los factores de la producción y de las pruebas a las que se sometió el modelo. Los datos de infraestructura del puerto representan muy bien a los insumos del capital $(\mathrm{K})$, en este caso, se considera a la longitud de muelle medido en metros. En cuanto al insumo trabajo (L), se contempla la cantidad de trabajadores de la terminal de contenedores de cada puerto. Por lo que se refiere al output, se usa el número de teus manejados anualmente en cada puerto de la región del APEC.

La disponibilidad de información puede llegar a ser una limitante para este tipo de análisis ya que las bases de datos por lo general vienen desagregadas por países y no por puertos. Sin embargo, para esta investigación se obtuvieron los datos del World Shipping Council y del Port Industry Statistics - American Association of Port Authorities, además de los sitios portuarios oficiales de cada uno de los puertos analizados.

Se presentan a continuación los estadísticos descriptivos de los años 2005 y 2015 (véase tablas 3 y 4), donde se muestran las medias y desviación estándar de las variables seleccionadas. En el caso de los teus y la longitud de muelle se observa un crecimiento en el periodo analizado. El número de trabajadores tiene un comportamiento diferente, ya que se presenta una disminución en la media durante el periodo debido principalmente al aumento del uso de maquinaria en las terminales de contenedores.

Tabla 3

Estadísticos descriptivos del año 2005

\begin{tabular}{llllll}
\hline Variable & Obs & Media & Desv. Est. & Min & Max \\
\hline Teus & 440 & 3.556 & 1.588013 & .103 & 23.192 \\
Long. Muelle & 440 & $3,366.00$ & 160.1709 & 550 & $6,114.00$ \\
Trabajadores & 440 & 175 & 40.4663 & 20 & 350 \\
\hline
\end{tabular}

Fuente: Elaboración propia con base en los resultados del estadístico SPSS.

Tabla 4

Estadísticos descriptivos del año 2015

\begin{tabular}{llllll}
\hline Variable & Obs & Media & Desv. Est. & Min & Max \\
\hline Teus & 440 & 5.197 & 7.588013 & .106 & 36.516 \\
Long. Muelle & 440 & $4,095.00$ & 160.1709 & 550 & $6,114.00$ \\
Trabajadores & 440 & 152 & 40.4663 & 15 & 310 \\
\hline
\end{tabular}

Fuente: Elaboración propia con base en los resultados del estadístico SPSS.

\section{Especificación del modelo de datos de panel}

El modelo de datos de panel lo conforman las 40 terminales de contenedores del APEC en un periodo de 11 años (2005-2015). 
Para determinar el tipo de modelo de datos de panel que se utilizará, se lleva a cabo el test de Haussman, donde se plantea que los estimadores de efectos aleatorios y de efectos fijos no difieren sustancialmente (se asume que los estimadores son los mismos). Por lo que la hipótesis nula es: no existe diferencia sistemática entre los coeficientes; si la prob chi2 >0.05, se acepta la hipótesis nula, lo que indica que el estimador aleatorio debe de ser utilizado, en caso contrario si Prob chi $2<0.05$, se rechaza la hipótesis nula y emplearíamos el estimador de efectos fijos.

Una vez que se llevó a cabo la prueba de Hausman (ver la tabla A1 del anexo), se observa que el valor Prob chi2 $0.1003>0.05$, por lo tanto se acepta la hipótesis nula y se selecciona el estimador de efectos variables.

Para Greene (2005), los análisis de frontera estocástica realizados con datos de panel se han basado en modelos tradicionales de efectos fijos y aleatorios. Greene propone extensiones que evitan dos deficiencias de estos enfoques: en primer lugar, los estimadores de datos de panel convencionales asumen que la ineficiencia técnica o de costos es invariante en el tiempo y en segundo, los estimadores de efectos fijos y aleatorios fuerzan en cualquier momento la heterogeneidad de unidades cruzadas invariantes en el mismo término que se está utilizando para capturar la ineficiencia.

La propuesta de Green (2005) para los modelos de datos panel, es separar el componente de heterogeneidad individual del componente de eficiencia técnica. Esta propuesta la llamó True Fixed Effects, para el caso del modelo de efectos fijos y True Random Effects para el modelo de efectos aleatorios. En estos modelos se incorpora una intersección específica para cada unidad de decisión y se puede obtener la ineficiencia variante en el tiempo.

En el trabajo se sigue una especificación translogarítmica para estimar la función de producción y así, obtener el componente del cambio tecnológico no neutral. Además, se aplica el modelo de efectos aleatorios propuesto por Greene (2005), porque se considera que en el periodo analizado es posible que el término de ineficiencia sea variable en el tiempo y por tanto, los efectos heterogéneos de cada puerto deben ser considerados en la estimación.

\section{Pruebas del modelo}

Una vez determinado el tipo de modelo de datos de panel con el que se va a estimar la frontera de producción, se continua con las demás pruebas que le dan la validez al modelo: especificación del modelo de Ramsey, la prueba White de heterocedasticidad, la prueba de multicolinealidad, la prueba de raíz unitaria, la prueba de cointegración y la prueba de asimetría.

\section{Prueba de Ramsey de especificación del modelo}

La prueba de especificación de Ramsey (1969), permite conocer si existe error en la especificación del modelo y detecta variables relevantes omitidas o irrelevantes incluidas. La 
hipótesis nula es que el modelo no tiene variables omitidas. En este caso después de haber hecho la prueba, se observa en la tabla A2 del anexo, que se obtuvo un valor $0.145>0.05$, y el valor $\mathrm{F}$ obtenido es de 1.23 siendo menor al valor $\mathrm{F}$ de la tabla, el cual tiene un valor crítico F de 1.927. Por lo que se acepta la hipótesis nula y se comprueba que no se ha omitido una variable relevante en el modelo.

\section{Prueba White de heterocedasticidad}

Existen diversas pruebas para detectar problemas de heterocedasticidad, uno de ellos es la prueba White, donde se plantea como hipótesis nula que no hay heterocedasticidad, es decir, que la varianza de los errores es constante. Una vez corrido el modelo, si el chi cuadrado obtenido es mayor al chi cuadrado de la tabla, se rechaza la hipótesis nula. (Gujarati y Porter, 2010). En los resultados obtenidos de esta prueba (ver tabla A3 del anexo), se observa que el valor de chi $2=12.01$, que es menor al valor de tablas, que en este caso es de 16.9190 al $5 \%$ para 9 grados de libertad, además se obtuvo un valor $\mathrm{p}$ value de $0.2357>0.05$. Por lo que cual se concluye que se acepta la hipótesis nula y se valida el supuesto de homocedasticidad.

\section{Prueba de multicolinealidad}

Esta prueba se realiza con la finalidad de verificar si el modelo tiene un alto grado de correlación. Existen diversas pruebas para detectar problemas de multicolinealidad, una de ellas es la prueba VIF (Variance Inflation Factor). Esta prueba calcula los datos de inflación de la varianza centrados o no centrados para las variables independientes especificadas en un modelo de regresión lineal (Gujarati y Porter, 2010). Los valores mayores que 10 se consideran indicativos de multicolinealidad. De acuerdo a los resultados de la tabla A 4 del anexo, se puede observar que no hay evidencia de multicolinealidad, al obtenerse un valor de 1.33 (Bruin, 2006).

\section{Análisis de estacionariedad}

En datos panel existen varias pruebas aceptadas por diversos autores para comprobar la estacionariedad y el orden de integración; una de ellas es la prueba de Levin, Lin y Chu (2002), que es la que se utiliza en la presente investigación. Esta prueba considera un parámetro autorregresivo común para todos los paneles, incluyendo un intercepto o un intercepto y tendencia. La hipótesis nula que maneja el test es: ho = los paneles tienen raíz unitaria y la hipótesis alternativa: ha $=$ los paneles son estacionarios.

El contraste se realizó para cada una de las variables: lny (logaritmo de y, que es la variable dependiente número de teus manejados anualmente), lnk (logaritmo de k que es la variable independiente longitud de muelle) y $\ln$ (logaritmo de 1 , que es la variable independiente del número de trabajadores). En una primera instancia se realizó el cálculo en niveles y sin 
tendencia, teniendo como resultado raíz unitaria, posteriormente se realiza el contraste con primeras diferencias y en este caso los paneles ya no presentaron raíz unitaria, por lo que tuvieron un orden de integración I(1), es decir fueron estacionarias en primeras diferencias (ver tabla A5 del anexo).

\section{Prueba de cointegración}

Una vez realizada la prueba de raíz unitaria, al obtener la estacionariedad de las variables en el mismo orden de integración I(1), se pretende observar si existe equilibrio a largo plazo, es decir si están cointegradas. Existen herramientas para examinar si las variables están cointegradas, una de ellas es la prueba de cointegración para datos panel desarrollada por Pedroni (2004), que consiste en analizar a los coeficientes asociados a las variables explicativas, donde los interceptos y tendencias pueden variar para cada unidad transversal, de esta manera indica que existe un vector de cointegración para cada unidad de análisis. En esta prueba, la hipótesis nula es que no existe cointegración frente a la hipótesis alternativa que si hay cointegración. De acuerdo a los resultados obtenidos (ver tabla A6 del anexo), se rechaza la hipótesis nula de no cointegración para las variables analizadas I(1) con un nivel de significancia al 5\%; por lo que se concluye que todos los paneles están cointegrados y que existe un equilibro a largo plazo.

\section{Prueba de asimetría (Skeness)}

La prueba skeness sirve para especificar si se puede medir la ineficiencia en el modelo de frontera estocástica, en este caso, el signo negativo muestra que los residuales están ajustados correctamente para la implementación de máxima verosimilitud. En la frontera estocástica si el signo no es negativo entonces no podemos distinguir entre la ineficiencia y el error estocástico (Waldman, 1982). En la prueba (ver tabla A7 del anexo) el resultado del test de Skeness fue de -0.5605371 , obteniéndose el signo esperado.

\section{Modelo de función de producción}

A continuación se muestran los resultados del modelo de función de producción utilizando la estimación de máxima verosimilitud (véase tabla 5). 
Tabla 5

Modelo de función de producción SFA

\begin{tabular}{|c|c|c|c|c|c|}
\hline & Stoc. Frontier no & half model & Number of & & 440 \\
\hline & & & Wald chi2 & $=$ & 229.75 \\
\hline & & & Prob $>$ chi 2 & $=$ & 0.000 \\
\hline $\ln Y$ & Coef. & Std. Err. & $\mathrm{z}$ & $\mathrm{P}>\mathrm{z}$ & \\
\hline $\ln L$ & 0.557099 & 0.0257557 & 21.63 & 0.000 & \\
\hline $\ln K$ & 0.6358347 & 0.0343544 & 18.51 & 0.000 & \\
\hline $\ln L 2$ & -0.0537163 & 0.0119536 & -4.49 & 0.001 & \\
\hline $\ln K 2$ & -0.0850208 & 0.0147142 & -5.78 & 0.002 & \\
\hline $\mathrm{t} 2$ & 0.0199149 & 0.0019572 & 10.18 & 0.007 & \\
\hline $\operatorname{lnLK}$ & 0.1677688 & 0.0233009 & 7.2 & 0.045 & \\
\hline _cons & 0.4816761 & 0.0205502 & 23.44 & 0.004 & \\
\hline$/ \operatorname{lnsig} 2 \mathrm{v}$ & -7.110676 & 0.5478525 & -12.98 & 0.000 & \\
\hline$/ \operatorname{lnsig} 2 \mathrm{u}$ & -3.609345 & 0.2292352 & -15.75 & 0.000 & \\
\hline sigma_v & 0.6566085 & 0.0575254 & & & \\
\hline sigma_u & 0.99344928 & 0.1177844 & & & \\
\hline sigma2 & 1.418163 & 0.1824608 & & & \\
\hline lambda & 1.513067 & 0.1661375 & & & \\
\hline
\end{tabular}

Log Likelihood-ratio test of sigma_u= 0: chibar2 $(01)=12.16$ Prob $>$ chibar2 $=0.000$

Fuente: Elaboración propia con base en los cálculos realizados del estadístico STATA.

Como se puede observar en la tabla 5, los parámetros son diferentes a cero y el valor de lambda cercano a 2, lo que significa que el modelo de frontera estocástica es el adecuado para calcular la ineficiencia. Además el valor de sigma_u es muy cercano a uno, lo que indica que en el residuo se explica la ineficiencia en el modelo. Al final del cuadro 5 se encuentra el test de verosimilitud, el cual nos permite verificar si las restricciones son válidas o no. En este caso al rechazar la hipótesis nula está indicando que si son válidas las restricciones y que si se puede calcular con el modelo la ineficiencia. Todas estas pruebas en conjunto proporcionan la evidencia de que el modelo de datos de panel de efectos variables con la eficiencia variante en el tiempo puede utilizarse para poder estimar una frontera de producción, así como para 
realizar los cálculos correspondientes para el cambio de eficiencia técnica, cambio de eficiencia de escala, cambio tecnológico y el cambio en la Productividad Total de los Factores.

\section{Resultados}

Se observa en la tabla 6, que en promedio la PTF tuvo un valor en todo el periodo de estudio de 0.051 , lo que indica que hubo un incipiente crecimiento de la productividad de manera global en los puertos del APEC. Es el puerto de Lianyungung en China el que alcanza la productividad más alta, explicado esto, específicamente por el cambio tecnológico. En el lado opuesto, se encuentra el puerto de Lázaro Cárdenas en México con la menor productividad, donde el retroceso en el cambio tecnológico en los años de estudio es el que más incide en la caída de este indicador.

Tabla 6

Productividad Total de los Factores de los puertos del APEC, 2005-2015

\begin{tabular}{|c|c|c|c|c|}
\hline Puertos & $\begin{array}{c}\text { Cambio } \\
\text { Tecnológico }\end{array}$ & $\begin{array}{c}\text { Cambio } \\
\text { Eficiencia } \\
\text { Técnica }\end{array}$ & $\begin{array}{c}\text { Cambio } \\
\text { Eficiencia Escala }\end{array}$ & $\begin{array}{c}\text { Cambio } \\
\text { PTF }\end{array}$ \\
\hline Lianyungung, China & 0.448 & -0.064 & 0.042 & 0.425 \\
\hline Shanghai, China & 0.461 & -0.085 & 0.019 & 0.394 \\
\hline Okland- Sn Francisco, USA & 0.184 & -0.056 & 0.138 & 0.266 \\
\hline Suzhou, China & 0.255 & -0.062 & 0.036 & 0.229 \\
\hline Manzanillo, México & 0.156 & -0.027 & 0.015 & 0.144 \\
\hline Kwangyang, Corea & -0.028 & 0.006 & 0.143 & 0.121 \\
\hline Seattle-Tacoma, USA & 0.108 & -0.034 & 0.032 & 0.106 \\
\hline Ho Chi Minh, Vietnam & 0.059 & -0.020 & 0.051 & 0.090 \\
\hline Laem Chabang, Tailandia & 0.116 & -0.044 & 0.004 & 0.075 \\
\hline Guangzhou, China & 0.028 & -0.015 & 0.061 & 0.074 \\
\hline Xiamen, China & 0.016 & -0.010 & 0.048 & 0.054 \\
\hline Dalian, China & 0.043 & -0.019 & 0.029 & 0.053 \\
\hline Tianjin, China & -0.009 & -0.001 & 0.061 & 0.051 \\
\hline Yingkou, China & -0.002 & -0.003 & 0.053 & 0.049 \\
\hline Ningbo, China & -0.077 & 0.026 & 0.098 & 0.046 \\
\hline Qingdao, China & -0.019 & 0.004 & 0.056 & 0.041 \\
\hline Tanjung Pelepas, Malasia & 0.045 & -0.024 & 0.017 & 0.038 \\
\hline Keelung, Taiwám & 0.076 & -0.041 & -0.001 & 0.034 \\
\hline Nagoya, Japón & 0.000 & -0.005 & 0.037 & 0.032 \\
\hline
\end{tabular}




\begin{tabular}{|c|c|c|c|c|}
\hline Tanjung Perak, S. Indonesia & -0.015 & 0.002 & 0.044 & 0.031 \\
\hline Port Kelang, Malasia & 0.011 & -0.010 & 0.019 & 0.020 \\
\hline Busan, Corea & -0.049 & 0.011 & 0.055 & 0.017 \\
\hline Sidney, Australia & -0.024 & 0.004 & 0.035 & 0.015 \\
\hline Shenzhen, China & -0.063 & 0.024 & 0.054 & 0.015 \\
\hline Tokio, Japón & -0.077 & 0.022 & 0.068 & 0.014 \\
\hline Hong Kong, China & -0.041 & 0.013 & 0.039 & 0.010 \\
\hline Singapure & -0.012 & 0.000 & 0.022 & 0.010 \\
\hline Callao, Perú & -0.049 & 0.016 & 0.033 & -0.001 \\
\hline Metro Vancouver, Canadá & -0.028 & 0.006 & 0.019 & -0.002 \\
\hline Bankgok, Tailandia & -0.063 & 0.025 & 0.034 & -0.005 \\
\hline Melbourne, Australia & -0.065 & 0.022 & 0.037 & -0.006 \\
\hline Kaohsiung, Taiwán & -0.041 & 0.012 & 0.021 & -0.008 \\
\hline Long Beach, USA & -0.068 & 0.027 & 0.030 & -0.012 \\
\hline Osaka, Japón & -0.103 & 0.027 & 0.060 & -0.017 \\
\hline Los Angeles, U.S.A. & -0.021 & 0.003 & -0.004 & -0.022 \\
\hline Manila, Filpinas & -0.080 & 0.028 & 0.026 & -0.025 \\
\hline Haiphong, Vietnam & -0.082 & 0.019 & 0.036 & -0.027 \\
\hline Tanjung Priok, J. Indonesia & -0.058 & 0.018 & -0.004 & -0.044 \\
\hline Inchon, Corea & -0.245 & 0.099 & 0.033 & -0.113 \\
\hline Lázaro Cárdenas, México & -0.199 & 0.040 & 0.016 & -0.143 \\
\hline Promedio & 0.012 & -0.002 & 0.040 & 0.051 \\
\hline
\end{tabular}

Fuente: Elaboración propia con base en los cálculos realizados con la metodología SFA.

En general en los puertos donde mayor problema se observa es en la eficiencia técnica, lo que da cuenta de la necesidad de optimizar mejor los recursos para aumentar esta eficiencia. Sin embargo, el puerto de Inchon en Corea es el que obtuvo el valor más alto en este indicador.

La eficiencia de escala en promedio tuvo un crecimiento positivo, lo que significa que los puertos están trabajando a una escala óptima, no necesariamente la deseada. Sobresale en este apartado el puerto Kwangyang en Corea quien presenta un aumento del 14.3\%. El cambio tecnológico en promedio refleja un aumento poco significativo, debido a que solo 14 puertos durante el periodo de estudio presentaron progreso tecnológico, sobresaliendo el puerto de Shangai con $46.10 \%$. Este puerto se encuentra administrado desde el año 2003, por el grupo Shanghai International Port Group (SIPG) quien sustituyó a la Autoridad del Puerto de Shanghái, logrando potencializarlo al recibir grandes inversiones para aumentar la 
infraestructura, equipo y facilitación en las operaciones portuarias (I.G, 2014).

En el caso de los puertos de México, es únicamente el de Manzanillo el que tuvo un crecimiento en su PTF, ello determinado principalmente por el cambio tecnológico. Esto explicado por las mejoras implementadas en la infraestructura y adquisición de maquinaria

en la terminal de contenedores a través del aumento de la inversión privada en los últimos años (API Manzanillo, 2018). En el lado opuesto, se encuentra el puerto de Lázaro Cárdenas con una caída en promedio de su PTF de -0.143 incidiendo en ello su retroceso tecnológico.

En general, se observa que la PTF de todos los puertos del APEC, se encuentra determinada en primer lugar por el aumento en la eficiencia de escala con un $4 \%$ y en segundo lugar por el progreso tecnológico de los puertos a nivel global con un 1.2\%, durante el periodo 2005-2015.

\section{Discusión de resultados}

Existen diversos autores que han estudiado la PTF en los puertos, pero son pocos los trabajos que lo han hecho instrumentando el Análisis de Frontera Estocástica como se realiza en el presente artículo.

Chang y Tovar (2014) calculan la eficiencia y la Productividad Total de los Factores de los puertos peruanos y chilenos a través del Análisis de Frontera Estocástica (SFA) en el periodo 2004-2010. Los resultados muestran que las terminales peruanas tuvieron un aumento en la PTF en promedio de $2.4 \%$, y se debió principalmente al aumento en la eficiencia técnica y de escala. En el caso de las terminales chilenas tuvieron una disminución en la PTF en promedio del $2.0 \%$ en los años de análisis, debido principalmente a las reducciones mostradas en el componente de cambio tecnológico. En este trabajo se tienen dos similitudes con respecto a estos autores: a) en ambas investigaciones se utiliza la metodología SFA implementando una función de producción translogarítmica; b) se desagrega también el cambio en la Productividad Total de los Factores en cambio tecnológico, cambio en la eficiencia técnica y cambio en la eficiencia de escala. Los resultados alcanzados se acercan más a los obtenidos en las terminales peruanas, ya que en esta investigación en los años analizados, se tuvo en promedio un crecimiento en la PTF del $5.1 \%$ originado principalmente por el cambio en la eficiencia de escala.

En la misma dirección De (2006) realizó un estudio sobre la Productividad Total de los Factores en los principales puertos de la India utilizando la metodología SFA, donde se concluye que el cambio tecnológico es el que más influye en la PTF. Dentro de las similitudes que se encuentran con los resultados de este autor es que en ambos trabajos se utiliza la metodología SFA. En las diferencias se tienen: a) en el trabajo de los puertos hindúes es el cambio tecnológico el que determina el crecimiento de la PTF, mientras que en este trabajo, es el aumento en la eficiencia de escala el que determina en mayor medida el aumento en la 
PTF; b) De (2006) utiliza una función de producción Cobb Douglas y en esta investigación se tiene una función translogarítmica; c) la PTF en los puertos de la India está desagregada en cambio tecnológico y cambio en la eficiencia técnica, en tanto que, en este estudio, además de estos dos componentes, se considera el cambio en la eficiencia de escala.

Lightfoot, Lubulwa y Malarz (2012), a través de métodos paramétricos calcularon la PTF mediante una función Cobb Douglas simple, así como también una función Cobb Douglas aumentada en función del tiempo, en los principales puertos de Australia durante el periodo 1997-2010. Las principales diferencias que se observan son: a) en estos autores se tiene un análisis de la PTF utilizando un modelo econométrico de mínimos cuadrados ordinarios con una función de producción Cobb Douglas, mientras que en este trabajo, se instrumenta un Análisis de Frontera Estocástica con una función de producción translogarítmica; b) ellos calculan la PTF de manera global. En este estudio, se desagrega el cálculo de la PTF en cambio tecnológico, cambio en la eficiencia técnica y cambio en la eficiencia de escala.

En resumen, en el presente trabajo, sobresalen tres aspectos: a) se realiza el estudio a través del Análisis de Frontera Estocástica utilizando datos de panel; b) la PTF se desagrega en cambio en la eficiencia técnica, cambio en la eficiencia de escala y cambio tecnológico; $\mathrm{y}, \mathrm{c})$ el aumento en la eficiencia de escala seguido por el cambio tecnológico son elementos esenciales en la determinación del crecimiento de la PTF. Constituyéndose en un trabajo pionero en las mediciones de la PTF a través de la metodología SFA en la temática portuaria.

\section{Conclusiones}

En este artículo se determinó la Productividad Total de los Factores (PTF) de 40 terminales de contenedores de los principales puertos de las economías miembros del Foro de Cooperación Económica Asia-Pacífico (APEC) durante el periodo 2005-2015. Se utiliza como variable dependiente los teus movilizados anualmente y como variables independientes longitud de muelle y personal ocupado. El modelo que se desarrolla es de datos de panel con efectos variables y se considera la función de producción translogarítmica propuesta por Kumbhakar y Lovell, (2004). Se obtiene así, el crecimiento de la PTF con cada uno de sus componentes: cambio tecnológico, cambio en la eficiencia y cambio en la eficiencia de escala, aplicando la metodología del Análisis de la Frontera Estocástica (SFA).

De los resultados se desprende que en promedio se tuvo un crecimiento en la productividad del $5.10 \%$, durante el período revisado y que fue el puerto de Lianyungung en China, el que alcanzó el nivel más alto en la PTF. Mientras que, el puerto de Lázaro Cárdenas tuvo la menor productividad para estos años. En materia de cambio tecnológico, es el puerto de Shangai en China, el que tuvo el mayor progreso tecnológico. En tanto que, en la eficiencia técnica, es 
el puerto de Inchon en Corea el que mejor se comportó en este indicador.

De los puertos mexicanos es el puerto de Manzanillo el que tuvo la PTF más alta (0.144) para los años de estudio y se encuentra determinada principalmente por el cambio tecnológico. Mientras que el puerto de Lázaro Cárdenas con una productividad de -0.143 , se ubica en el último lugar en las economías del APEC. La hipótesis planteada en este trabajo se cumple, ya que fue principalmente el cambio en la eficiencia de escala el que determinó la Productividad Total de los Factores en la región del APEC para el periodo 2005-2015.

Los resultados sugieren que el crecimiento de la PTF en los puertos del APEC puede beneficiarse especialmente de las políticas que promuevan la inversión en los insumos analizados en esta investigación: capital humano e infraestructura. Asimismo, los avances en materia de productividad al no ser significativos, sugieren la instrumentación de políticas públicas dirigidas a fortalecer la innovación y la tecnología, al ser este un indicador de poca incidencia en la determinación de la PTF en los puertos del APEC -más allá de que se hace necesario atender los problemas que se tienen en la eficiencia técnica-. Además de que, es la productividad un elemento de primer orden en el impacto económico y en el desarrollo de las economías que integran esta región.

Finalmente, se destacan tres aspectos de la presente investigación: a) se realiza el estudio a través del Análisis de Frontera Estocástica utilizando datos de panel en los puertos de la región del APEC durante el periodo 2005-2015; b) la PTF se desagrega en cambio en la eficiencia técnica, cambio en la eficiencia de escala y cambio tecnológico; y, c) el aumento en la eficiencia de escala seguido por el cambio tecnológico son elementos esenciales en la determinación del crecimiento de la PTF en los puertos del APEC. Constituyéndose en un trabajo pionero en las mediciones de la PTF a través de la metodología SFA en la temática portuaria.

\section{Referencias}

Afriat, S. N. (1972). Efficiency estimation of production functions. International Economic Review, 13(3), 568-598. DOI: 10.2307/2525845.

Aigner, D. J. y Chu, S. F. (1968). On estimating the industry production function. American Economic Review, 52(4), 762-782.

Aigner, D.J, Lovell, C. A. y Schmidt, P. (1977). Formulation and estimation of stochastic frontier production models. Journal of Econometrics, 6(1), 21-37. DOI: 10.1016/03044076(77)90052-5.

American Association of Port Authorities (AAPA)(2017). Port Industry Statistics. Disponible en: http://www.aapa-ports.org/unifying/content.aspx?ItemNumber=21048.Consultado $15 / 03 / 2018$.

Asia-Pacific Economic Cooperation (APEC) (2019). Transportation. Working Groups. 
Disponible en: https://www.apec.org/Groups/SOM-Steering-Committee-on-Economic-and-Technical-Cooperation/Working-Groups/Transportation. Consultado: 25/05/2019.

APEC (2016). Exploration on Strengthening of Maritime. Disponible en: Connectivityfile://C:/Users/Dell/Downloads/216TPTExploration\%20on\%20Strengthening\%20Maritime\%20Connecti\%20(1).pdf Consultado: 20/08/2018

-APEC (2015). Statistics APEC, Key Indicators Database. Disponible en: http:// statistics.apec.org/index.php/apec_psu/index Consultado el 18/03/2018

APEC (1989). 1989 APEC Ministerial Meeting. Recuperado de https://www. apec.org/Meeting-Papers/Annual-Ministerial-Meetings/1989/1989_amm Consultado: 10/02/2018.

API Manzanillo (2018). Puerto de Manzanillo. Disponible en https://www.puertomanzanillo.com.mx/espi/0000001/inicio, Consultado: 1/04/2018.

Baran, J. y Górecka, A. (2015). Seaport efficiency and productivity based on Data Envelopment Analysis and Malmquist Productivity Index. Logistics \& Sustainable Transport, Vol. 6, No. 1,-33, DOI: 10.1515/j1st-2015-0008

Batesse, G. E. y Coelli, T. J. (1988). Prediction of Firm Level Technical Efficiencies with a Generalized Frontier Production Function and Panel Data. Journal of Econometrics, 38, 387-399. DOI: https://doi.org/10.1016/0304-4076(88)90053-X

Battese, G.E. and Coelli, T.J. (1992) Frontier Production Functions, Technical Efficiency and Panel Data: With Application to Paddy Farmers in India. Journal of Productivity Analysis, 3, 153-169. DOI: https://doi.org/10.1007/BF00158774

Bofarull, M. (2010). El Sistema Portuario Español: Regulación, Entorno Competitivo y Resultados. Una Aplicación del Análisis Envolvente de Datos, Universitat Rovira I Virgili. Disponible en file:///C:/Users/Dell/Downloads/Memoria.pdf, Consultado: 20/09/2017.

Bruin, J. 2006. Newtest: command to compute new test. UCLA: Statistical Consulting Group. Disponible e: http://www.ats.ucla.edu/stat/stata/ado/analysis/. Consultado: 21/10/2018

Cardarelli. R y Lusinyan, L. (2015). U.S. Total Factor Productivity Slowdown: Evidence from the U.S. States. IMF Working Paper, WP/15/116

Caves, D.; Christensen, L. y W.E. Diewert (1982). The Economic Theory of Index Numbers and the Measurement of Input, Output and Productivity. Econometrica, 50 (6), 13931414.DOI: $10.2307 / 1913388$.

Chang y Tovar (2014); Efficiency and productivity changes for Peruvian and Chilean ports terminals:A parametric distance functions approach, Transport Policy, (31), 83-94. DOI: https://doi.org/10.1016/j.tranpol.2013.11.007

Chen, E. K. (1997). The Total Factor Productivity Debate: Determinants of Economic Growth in East Asia. Asian-Pacific Economic Literature, 10, 18-38. DOI: https://doi. org/10.1111/1467-8411.00002 
Cheon, S., Dowall, D. E., y Song, D. W. (2009). Typology of long-term port efficiency improvement paths: Malmquist total factor productivity for world container ports. Journal of Infrastructure Systems, 15(4), 340-350. DOI: https://doi.org/10.1061/(ASCE)10760342(2009)15:4(340)

Chun-Chu L. (2008) Evaluating the operational efficiency of major ports in the Asia-Pacific region using data envelopment analysis, Applied Economics, 40:13, 1737-1743, DOI: 10.1080/00036840600905126

Coelli, T. J., Prasada, D., O’Donnell, C., y Battese, G.E. (2005). An Introduction to Efficiency and Productivity Analysis. Estados Unidos: Springer Segunda Edición.

Cornwell, C., Schmidt, P., y Sickles, R. (1990). Production frontiers with cross-sectional and time-series variation in efficiency levels. Journal of Econometrics 46, 185-200. DOI: https://doi.org/10.1016/0304-4076(90)90054-W

De, P. (2006). Total Factor Productivity Growth: Indian Ports in the Era of Globalisation. Maritime Economics \& Logistics, 8 (4), 366-386. DOI: https://doi.org/10.1057/palgrave. mel.9100164

Diewert, W.E. (1992). The Measurement of Productivity. Bulletin of Economic Research, 44 (3), 163-198. DOI: https://doi.org/10.1111/j.1467-8586.1992.tb00542.x

Ding, Z., Jo, G., Wang, Y. y Yeo, G. (2015), The Relative Efficiency of Container Terminals in Small and Medium-Sized Ports in China. The Asian Journal of Shipping and Logistics. 31, 231-251. DOI: https://doi.org/10.1016/j.ajsl.2015.06.004

Dirección General de Puertos (2016). Objetivos Estratégicos. Coordinación General de Puertos y Marina Mercante. Disponible en: https://www.gob.mx/puertosymarinamercante/acciones-y-programas/objetivos-estrategicos-57912 Consultado: 29/03/2018

Evans, C. (1992). Productivity Shocks and Real Business Cycles. Journal of Monetary Economics, 29, 191-208. DOI: https://doi.org/10.1016/0304-3932(92)90012-Q

Färe, R., Grosskopf, S. y Roos P. (1998) Malmquist Productivity Indices: A Survey of Theory and Practice, in: R. Färe, S. Grosskopf, R. Russell (eds) Index Numbers: Essays in Honour of Sten Malmquist, Kluwer, Boston, 127-190.

Fu, B., Song, X. y Guo, Z. (2009). DEA-based Malmquist Productivity Index Measure of Operating Efficiencies: New Insights with an Application to Container Ports. Journal of Shanghai Jiaotong University (Science) 14, 490. DOI: https://doi.org/10.1007/s12204009-0490-8.

García, S. (2018). Las plataformas logísticas y los nodos de articulación modal. Aduanas revista, Eje del Comercio Exterior, publicado el 20 de septiembre de 2018 Editorial Aduanas. Disponible en: http://aduanasrevista.mx/las-plataformas-logisticas-y-los-nodos-de-articulacion-modal-2/ Consultado: el 20/junio/2019

Giraleas, D. (2013). The measurement and decomposition of economy-wide productivity growth. Assessing the accuracy and selecting between different approaches. Tesis Doctoral, Aston University, UK 
González, M. y L.Trujillo, (2006). La Medición de la Eficiencia en el Sector Portuario: Revisión de la Evidencia Empírica. Canarias, España

Greene, W. (2005). Fixed and Random Effects in Stochastic Frontier Models. Journal of Productivity Analysis, Springer, 23(1), pp 7-32. DOI: 10.1007/s11123-004-8545-1

Gujarati, D. y Porter, D. (2010). Econometría, 5ta edición. México D.F.: Editorial Mc Graw Hill.

Halkos, G. y Tzeremes, N. (2012). Measuring Seaports' Productivity: A Malmquist Productivity Index Decomposition Approach. MPRA Paper No. 40174, posted 20. Disponible en: https://mpra.ub.uni-muenchen.de/40174/1/MPRA_paper_40174.pdf, Consultado: 20/ 10/ 2017.

Hall, R. (1988). The relation between Price and Marginal Cost in US Industry. Journal of Political Economy, pp. 921-947. DOI http://dx.doi.org/10.1086/261570

Hall, R. (1990). Invariance Properties of Solow's Productivity Residual, in P. Diamond,ed., Growth, Productivity and Employment, Cambridge: The MIT Press, pp. 71-112.

Hsuan-Shih, L. y Ming-Tao, Ch. (2005), A Fuzzy Multiple Criteria Decision Making Model for Airline Competitiveness Evaluation, Conference: Knowledge-Based Intelligent Information and Engineering Systems, 10th International Conference, KES 2006, Bournemouth, UK, October 9-11, 2006, Proceedings, Part II. DOI: 10.1007/11893004_115

Ingeniería por el Mundo (IG) (2014). Puerto de Shangai. Disponible en: http://ingenieriaporelmundo.blogspot.mx/2014/10/puerto-de-shanghai.html Consultado: 25/03/ 2018

International Maritime Organization (IMO) (2015). Maritime Industry of Asia Pacific. Disponible en http://www.imo.org/en/About/Pages/Default.aspx. Consultado: 11/02/ 2016

Jondrow, J. Lovell, K., Materov I. y Schmidt, P. (1982). On the estimation of technical inefficiency in the stochastic frontier production function model. Journal of Econometrics, 19, 233-238. DOI: https://doi.org/10.1016/0304-4076(82)90004-5

Kennedy, O. R., Lin, K., Yang, H., and Ruth, B. (2011). Sea-port Operational Efficiency: An Evaluation of Five Asian Ports Using Stochastic Frontier Production Function Model. Journal of Service Science and Management 4(3), 391-399. DOI: 10.4236/ jssm.2011.43045

Kim, M. y A. Sachis, (1986). The Structure of Production, Technical Change and Productivity in a Port. International Journal of Industrial Economics, 35 (2), 209-223. DOI: $10.2307 / 2098359$

Kumbhakar, S. C. (1990) Production Frontiers, Panel Data, and Time-Varying Technical Inefficiency. Journal of Econometrics, 46, 201-12. DOI: https://doi.org/10.1016/0304-4076(90)90055-X

Kumbhakar, S., y Lovell K. (2000) Stochastic Frontier Analysis. Cambridge University Press, Cambridge

Kumbhakar, S. y Lovell, K. (2004). Stochastic Frontier Analysis. Cambridge University Press, Cambridge 
Kumtong, Saosaovaphak y Chaiboonsri (2017). Measuring the total factor productivity for international seaport in South East Asia (ASEAN). Journal of Advanced Research in Social Sciences and Humanities, 2 (3), 154-167. DOI: https://dx.doi.org/10.26500/JARSSH-02-2017-0301

Kutin, Thuy y Valléec (2017), Relative Efficiencies of ASEAN Container Ports based on Data Envelopment Analysis. The Asian Journal of Shipping and Logistics, 33 (2), pp. 67-77. DOI: https://doi.org/10.1016/j.ajs1.2017.06.004

Levin, A., Lin C. y Chu, C. (2002). Unit root tests in panel data: Asymptotic and finite-sample properties. Journal of Econometrics, 108,1-24. DOI: https://doi.org/10.1016/S03044076(01)00098-7

Lightfoot, A.; Lubulwa, G., y Malarz, A. (2012). An analysis of container handling at Australian ports en 35th ATRF Conference 2012, Perth, Western Australia. Disponible http:// atrf.info/papers/2012/2012_Lightfoot_Lubulwa_Malarz.pdf. Consultado: 10/01/2018.

Mankiw, N. G. (1989). Real Business Cycles: A New Keynesian Perspective. Journal of Economic Perspectives, 3, 79-90.

Meeusen, W. y Van den Broeck, J. (1977). Efficiency estimation from Cobb-Douglas production functions with composed error. International Economic Review, 18(2), 435-444. DOI: $10.2307 / 2525757$

Mokhtar, H. y Shah, (2013). Malmquist Productivity Index for Container Terminal. European Journal of Business and Management, 5 (2), 58-71

Nwanosike, F.; Tipi, N. y Warnock-Smith, D., (2016). Productivity change in Nigerian seaports after reform: a Malmquist productivity index decomposition approach. Maritime Policy \& Management. 43(7), 798-811. DOI: https://doi.org/10.1080/03088839.2016.11 83827

Park, N. (2010). A Study on Productivity Factors of Chinese Container Terminals, Journal of Navigation and Port Research International Edition, 34 (7), 559-566. DOI: 10.5394/ KINPR.2010.34.7.559

Pedroni, P. (2004). Panel cointegration: Asymptotic and finite sample properties of pooled time series tests with an application to the PPP hypothesis. Econometric Theory, 20(3), 597-625. DOI: https://doi.org/10.1016/0304-3878(81)90004-3

Pitt, M. y Lee, L. (1981). The measurement and sources of technical inefficiency in the Indonesian weaving industry. Journal of Development Economics, 9, 43-64. DOI: https:// doi.org/10.1016/0304-3878(81)90004-3

Ramsey, J. (1969). Test for Specification Errors in Classical Linear Least Square Regression Analysis. Journal of the Royal Statistical Society. Series B, 31(2), 350-371

Richmond, J. (1974). Estimating the Efficiency of Production. International. Economic Review, 15, 515-521. DOI: 10.2307/2525875

Sánchez, L. y Mouftier, L. (2016). Reflexiones sobre el futuro de los puertos. División de recursos naturales e infraestructura CEPAL. Boletín Marítimo No. 64, noviembre 2016. 
Sánchez, R., Jaimurzina, A., Wilmsmeier, G., Pérez G., Doer, O.,y Pinto, F., (2015). Transporte marítimo y puertos. Desafíos y oportunidades en busca de un desarrollo sostenible en América Latina y el Caribe (2015)

Schmidt, P.y Sickles, R. (1984). Production frontiers and Panel Data. Journal of Business and Economic Statistics 2(4/October), 367-374. DOI: 10.2307/1391278

Schøyen, H. y Odeck, J. (2017). Comparing the productivity of Norwegian and some Nordic and UK container ports - an application of Malmquist productivity index. Int. J. Shipping and Transport Logistics. 9 (2), 234-256.

Seitz, W. D. (1971). Productive Efficiency in the Steam-Electric Generating Industry. Journal of Political Economy, 79, 878-886.

Solow, R. (1957). Technical Change and the Aggregate Production Function. The MIT Press, 39, 312-320. DOI: 10.2307/1926047.

Songqing J., Hengyun, M., Jikun H., Ruifa H., y Rozelle, S. (2009). Productivity, efficiency and technical change: measuring the performance of China's transforming agriculture. Journal of Productivity Analysis, Springer, 33(3), pp. 191-207. DOI: 10.1007/s11123009-0145-7

Sumanth, D. (1994). Ingenieria y Administración de la Productividad; medición, evaluación, planeación y mejoramiento de la productividad en las organizaciones de manufactura y servicio. México: Mc Graw-Hill.

Summers, L. (1986). Some Skeptical Observations on Real Business Cycle Theory. Federal Reserve Bank of Minneapolis Quarterly Review 10 (4), 22-26.

Timmer, C. P. (1971). Using a Probabilistic Frontier Production Function to Measure Technical Efficiency. Journal of Political Economy 79, 776-794.

United Nations Conference on Trade and Development (UNCTAD) (2018). Review of Maritime Transport 2018. Disponible en https://unctad.org/en/PublicationsLibrary/ rmt2018_en.pdf

United Nations Conference on Trade and Development (UNCTAD) (2018). Review of Maritime Transport 2018. Disponible en https://unctad.org/en/PublicationsLibrary/ rmt2018_en.pdf Consultado 20/09/2019

United Nations Conference on Trade and Development (UNCTAD) (2017). Review of Maritime Transport 2017. Disponible en https://unctad.org/en/PublicationsLibrary/ rmt2017_en.pdf Consultado: el 15/06/2019

United Nations Conference on Trade and Development (UNCTAD) (2016). Review of Maritime Transport 2016. Disponible en https://unctad.org/en/PublicationsLibrary/ rmt2016_en.pdf Consultado: el 15/06/2019

United Nations Conference on Trade and Development (UNCTAD) (2015). Review of Maritime Transport 2015. Disponible en https://unctad.org/en/PublicationsLibrary/ rmt2015_en.pdf Consultado: el 15/06/2019

Waldman, D. (1982). A Stationary Point for Stochastic Frontier Likelihood. Journal of Econometrics. 18, 275-279. DOI: https://doi.org/10.1016/0304-4076(82)90041-0 
World Shipping Council (2017). Top 50 World Container Ports. Disponible en http://www. worldshipping.org/about-the-industry/global-trade/top-50-world-container-ports Consultado: 2/01/2018.

World Economic Forum (WEF) (2017). The Global Competitiveness Report 2017-2018. Disponible http://www3.weforum.org/docs/GCR2016- 2017/05FullReport/TheGlobalCompetitivenessReport2016-2017_FINAL.pdf Consultado: 15/01/2018

Yen-Chun, Chih-Hung Yuan, M. Goh y Yung-Hsiang Lu (2016). Regional Port Productivity in APEC. Sustainability, 8(7), 689. DOI: https://doi.org/10.3390/su8070689

Zheng, J., Liu, X., y Bigsten, A. (1998), Ownership Structure and Determinants of Technical Efficiency: An Application of Data Envelopment Analysis to Chinese Enterprises (1986-1990). Journal of Comparative Economics 26 (3), 465-484. DOI: https://doi. org/10.1006/jcec.1998.1540

\section{Anexo}

Tabla A1

Prueba de Hausman

\begin{tabular}{ll}
\hline hausman fixed random \\
\hline Test: $\quad$ Ho: difference in coefficients not systematic \\
chi2(2) $=\quad 4.6$ \\
Prob $>$ chi2 $=\quad 0.1003$ \\
\hline
\end{tabular}

Fuente: Elaboración propia con base en los resultados del estadístico Stata

Tabla A2

Prueba de especificación

\begin{tabular}{|c|c|}
\hline \multicolumn{2}{|c|}{ Modelo Ramsey } \\
\hline estat ovtest & \\
\hline \multicolumn{2}{|c|}{ Ho: model has no on } \\
\hline $\mathrm{F}(9,329)=$ & 1.23 \\
\hline Prob $>F=$ & 0.14 \\
\hline
\end{tabular}

Fuente: Elaboración propia con base en los resultados del estadístico Stata 
Tabla A3

Prueba de heterocedasticidad

White's test for Ho: homoskedasticity

against Ha: unrestricted heteroskedasticity

$\operatorname{chi} 2(9) \quad=12.01$

Prob > chi $2=0.2357$

Fuente: Elaboración propia con base en los cálculos realizados del estadístico STATA

Tabla 4

Prueba VIF

\begin{tabular}{lcc}
\hline Multicolinealidad & & \\
\hline Variable & VIF & $1 /$ VIF \\
lny & 1.5 & 0.6655 \\
\hline $\operatorname{lnk}$ & 1.42 & 0.7023 \\
$\mathrm{t}$ & 1.07 & 0.9365 \\
Mean VIF & 1.33 & \\
\hline
\end{tabular}

Fuente: Elaboración propia con base en los cálculos realizados del estadístico STATA

Tabla A5

Prueba de raíz unitaria Levin-Lin-Chu

Ho: Panels contain unit roots Number of panels $=40$

Ha: Panels are stationary Number of periods $=10$

$\begin{array}{lll}\text { Variable } & \text { Statistic } & \text { p-value } \\ \text { D.lny } & -39.4048 & 0.0000 \\ \text { D.lnL } & .7 .3817 & 0.0000 \\ \text { D.Lnk } & -20.2809 & 0.0000\end{array}$

Fuente: Elaboración propia con base en los cálculos realizados del estadístico STATA. 
O. V. Delfín-Ortega y C. L. Navarro-Chávez / Contaduría y Administración 66(1) 2021, 1-34 http://dx.doi.org/10.22201/fca.24488410e.2021.1998

Tabla A6

Prueba de cointegración Pedroni

. xtcointtest pedroni lny $\operatorname{lnl} \operatorname{lnk}$

Ho: No cointegration

Number of panels $=40$

Ha: All Panels are cointegrated

Number of periods $=10$

\begin{tabular}{lcc}
\hline & Statistic & p-value \\
Modified Phillips-Perron t & -7.3452 & 0.001 \\
Phillips-Perron t & -5.8764 & 0.023 \\
Augmented Dickey-Fuller t & -5.3243 & 0.000 \\
\hline
\end{tabular}

Fuente: Elaboración propia con base en los cálculos realizados del estadístico STATA

\section{Tabla A7}

Prueba de asimetría (skewness)

\begin{tabular}{lc}
\hline Skewness & -0.5605371 \\
Variance & 0.798205 \\
Kurtosis & 5.089777 \\
\hline
\end{tabular}

Fuente: Elaboración propia con base en los cálculos realizados del estadístico STATA. 